\title{
A Decentralized Routing Control Scheme for Data Communication Networks
}

\author{
Xueming Si, ${ }^{1}$ Xun-Lin Zhu, ${ }^{2}$ Xin Du, ${ }^{3}$ and Xiangpeng Xie ${ }^{4}$ \\ ${ }^{1}$ State Key Laboratory of Mathematical Engineering and Advanced Computing, Zhengzhou 450002, China \\ ${ }^{2}$ Department of Mathematics, Zhengzhou University, Zhengzhou 450001, China \\ ${ }^{3}$ School of Mechatronic Engineering and Automation, Shanghai University, Shanghai 200444, China \\ ${ }^{4}$ Department of Control Science and Engineering, Huazhong University of Science and Technology, Wuhan 430074, China
}

Correspondence should be addressed to Xun-Lin Zhu; xlzhu@zzu.edu.cn

Received 11 January 2013; Accepted 4 March 2013

Academic Editor: Engang Tian

Copyright (c) 2013 Xueming Si et al. This is an open access article distributed under the Creative Commons Attribution License, which permits unrestricted use, distribution, and reproduction in any medium, provided the original work is properly cited.

\begin{abstract}
This paper studies the decentralized routing control of data communication networks. Taking the physical constraints on the data flow and the network-induced delays into consideration, the decentralized routing control problem is reformulated as a constrained control problem, and the restriction of initial message lengths is no longer needed. By defining a general Lyapunov functional and applying a reciprocally convex combination technique to deal with the network-induced delays, less conservative and less complex designing conditions are obtained in terms of linear matrix inequalities (LMIs). A simulation example is also given to illustrate the effectiveness of the proposed designing scheme.
\end{abstract}

\section{Introduction}

Routing control plays an indispensable role in complex and crowded networks such as transportation networks and data communication networks [1-19]. The fundamental idea of routing control is that the most suitable route for data packets from source to destination through other nodes is to be determined such that certain objective functions are minimized.

Early routing algorithms, such as those implemented in the ARPANET [2] and TYMNET [3], were designed for optimally achieving some prespecified objective, such as shortest time or shortest path from the initial node to the destination node, minimization of a cost function related to the link congestion [14], or minimization of a generic measure of link flows. However, the performance of the crowded networks with these routing algorithms will deteriorate significantly, since the channel communication capacity is usually limited and the link congestion problems may often occur in many practical networks [20]. Therefore, it is essential to take the physical constraints, such as limited channel capacity and limited energy source, into consideration in designing proper routing control scheme.
It is very known that the network-induced delays (i.e., transmission delay, propagation delay, and processing delay) may induce instability, oscillation, and poor performance, and have a major impact on the routing control design. Several researchers have also attempted to address the problem of delay-constrained routing in recent years [13-19, 21-24]. Different techniques such as capacity allocation [16] and neural networks [19] are adopted to obtain the proper routing paths that minimizing or guaranteeing the boundedness of different types of network delays. However, more general index may be specified in evaluating the network performance, and many physical constraints of networks also should be taken into consideration. Under such a circumstances, the routing control problem becomes a difficult task and cannot be tackled with the above-mentioned methods. As stated in $[18,19]$, the fluid flow conservation principle is frequently employed in deriving the queuing dynamics, wherein each state of the subsystem (node) represents a queue corresponding to a given destination node. With the aid of linear matrix inequality (LMI) approach, the recent paper [20] provides a promising framework to revisit and reformulate the routing control problems as a constrained $H_{\infty}$ optimal control problem for time-delay systems; hence, a number of 
physical constraints can be easily imposed on the routing problem.

In [20], a continuous-time system model was used to describe the dynamics of traffic networks, and the physical constraints on traffic flows and queues were transformed into a set of LMI-based conditions. Then, robust centralized as well as decentralized routing control strategies were introduced for networks with a fixed topology based on the minimisation of the worst-case queuing length, which is related to the queuing delays. Similar treatment was adopted in [25]. It is worth pointing out that the derivatives of network-induced delays were assumed to be less than 1. However, according to [26], such assumptions are false. Meanwhile, a parameter should be given a priority and the initial values of all message queue lengths are required to be 0 in the transformation of the physical constraints, which may be harsh for many practical applications and may result in some conservatism.

In this paper, we study routing control of data communication networks that arise from many practical applications [19]. A discrete-time model instead of continuous-time model [20] is used to describe the communication network dynamics and the data constraints on the data flow. Furthermore, in order to facilitate the practical implementation for large-scale data communication networks, we are dedicated to design decentralized controllers instead of centralized controllers. Thus, vulnerable failures and large communication overheads in the network may be avoided. To this end, some new techniques will be adopted to tackle the extra difficulty in the related LMI manipulation and derivation, which are caused because of the decentralized controller structure. Different from the existing works, a reciprocally convex combination method is used to deal with delays, the physical constraints are successfully transformed into a set of LMIs and the restriction that initial message queue lengths should be 0 is removed, and each LMI-based condition involved in the designing of decentralized routing control is only related to an oriented link in the data communication network, which can effectively reduce both the conservatism and the complexity.

Notation. Throughout this paper, a real symmetric matrix $P>0(\geq 0)$ denotes $P$ being a positive definite (positive semidefinite) matrix, and $A>B(A \geq B)$ means $A-B>$ $0(\geq 0) . I$ is used to denote an identity matrix with proper dimension. Matrices, if not explicitly stated, are assumed to have compatible dimensions. The symmetric terms in a symmetric matrix are denoted by $*$. For a real vector $x \in R^{n}$, $x \geq 0(>0)$ denotes every component of $x$ being nonnegative (positive). In this case, $x$ is called nonnegative (positive). Likewise, $A \in R^{n \times m}$ is nonnegative or positive if every entry of $A$ is nonnegative or positive, respectively, which is written as $A \geq 0$ or $A>0$, respectively.

\section{Problem Formulation}

Consider a data communication network, $\mathscr{G}=(\mathscr{N}, \mathscr{L})$, consisting of a directed graph with a set $\mathscr{N}$ of $N$ nodes and a set $\mathscr{L}$ of $L$-oriented links. Each message has a destination node $d \in \mathcal{N}$, and all destination nodes form a set $\mathcal{N}_{d}$. Each node $i \in \mathcal{N}$ receives messages from the upstream nodes within the network. Messages are absorbed as soon as they arrive at their destination nodes. Messages arriving to a node other than their final destination are put into a queue (or buffer) and eventually are sent out to a downstream node. It is assumed that the network is "connected," that is, each node of the network must be reachable from each other node. In the worst case, where all the nodes are source as well as destination, at each node $i \in \mathcal{N}$ there will be $n-1$ queues in which messages are stored for all destinations, $1,2, \ldots, i-$ $1, i+1, \ldots, N$. We also assume that the rate of messages that are sent from one node to another is updated (synchronously throughout the communication network) by control variables at discrete instants $k T(k=0,1,2, \ldots)$, where $T$ is a positive scalar.

Similar to [19], the data communication network dynamics can be expressed as

$$
\begin{array}{r}
q_{i}^{d}(k+1)=q_{i}^{d}(k)+\sum_{\substack{j \in \mathscr{P}(i) \\
j \neq d}} f_{j i}^{d}\left(k-\tau_{j i}(k)\right)-\sum_{j \in \mathcal{S}(i)} f_{i j}^{d}(k), \\
i \in \mathcal{N}, d \in \mathcal{N}_{d}^{i},
\end{array}
$$

where $q_{i}^{d}(k)$ : message queue length at node $i$ destined to node $d$ at instant $k T ; \mathscr{P}(i)$ : set of upstream neighbors of node $i$; $\mathcal{S}(i)$ : set of downstream neighbors of node $i ; f_{i j}^{d}(k)$ : traffic flow routed from node $i$ to node $j$ destined to node $d$ at instant $k T ; \mathscr{N}_{d}^{i}$ : subset of $\mathcal{N}_{d}$, that is, $\mathcal{N}_{d}^{i}=\mathcal{N}_{d} \backslash\{i\} ; \tau_{i j}(k)$ : timevarying delay in transmitting, propagating, and processing of messages (including identifying the destination, inserting in the queue, and routing computation) with destination $d$ routed from node $i$ to node $j$, which satisfies

$$
0 \leq \tau_{i j}(k) \leq \tau_{i j}, \quad \forall(i, j) \in \mathscr{L},
$$

where $\tau_{i j}$ are constants.

Due to the physical significance of $f_{i j}^{d}$ and the constraints on the data flow through each link and the message queue buffer size at each node, the following conditions should be satisfied:

$$
\begin{gathered}
f_{i j}^{d}(k) \geq 0, \quad i \in \mathcal{N}, j \in \mathcal{S}(i), d \in \mathcal{N}_{d}^{i}, \\
0 \leq \sum_{d \in \mathcal{N}_{d}^{i}} q_{i}^{d}(k) \leq q_{i}, \quad i \in \mathcal{N}, \\
\sum_{d \in \mathcal{N}_{d}^{i}} f_{i j}^{d}(k) \leq c_{i j}, \quad i \in \mathcal{N}, j \in \mathcal{S}(i),
\end{gathered}
$$

where positive scalars $q_{i}$ and $c_{i j}$ represent the maximal length of the message queue length at node $i$ and the maximal capacity of oriented link $(i, j)$ (it is also called "edge"), respectively.

Define

$$
\begin{gathered}
x_{i}(k)=\operatorname{vec}\left\{q_{i}^{d}(k)\right\}, \quad u_{i}(k)=\operatorname{vec}\left\{f_{i j}^{d}(k)\right\}, \\
\left(i \in \mathcal{N}, j \in \mathcal{S}(i), d \in \mathcal{N}_{d}^{i}\right),
\end{gathered}
$$


then (1) can be rewritten as

$$
x_{i}(k+1)=x_{i}(k)+B_{i} u_{i}(k)+\sum_{j \in \mathscr{P}(i)} B_{d j i} u_{j}\left(k-\tau_{j i}(k)\right),
$$

where $x_{i}(k) \in R^{N_{d}^{i}}$ and $u_{i}(k) \in R^{m_{i} N_{d}^{i}}$ are vectors; $m_{i}, N_{d}$, and $N_{d}^{i}$ represent the numbers of nodes contained in $\delta(i), \mathcal{N}_{d}$, and $\mathcal{N}_{d}^{i}$, respectively; $B_{i}$ and $B_{d j i}$ are constant matrices. Obviously, the entries of $B_{i}$ are equal to 0 or -1 , and the ones of $B_{d j i}$ are equal to 0 or 1 , which reflect the network connectivity of node $i$ in graph $\mathscr{G}$ [20].

In this paper, a decentralized routing control scheme

$$
u_{i}(k)=K_{i} x_{i}(k), \quad i \in \mathcal{N},
$$

is proposed to stabilize the origin system (1), where state feedback controllers $K_{i}(i \in \mathcal{N})$ are to be designed. Such a constrained control problem can be found by solving a set of linear matrix inequalities (LMIs). Different from the one given in [20], the obtained routing control scheme is at edge-level; that is, each inequality is only dependent on an edge $(i, j)$ in $\mathscr{L}$. This implies that the dimension of each inequality is low, so the computational complexity is reduced effectively. In addition, since some constraints are discarded, the conservatism of the obtained LMI-based conditions is also reduced.

Through this paper, Jensen's integral inequality [27] and a reciprocally convex combination technique [28] will be used, so it is listed as the following lemmas.

Lemma 1 (see [27]). For any positive symmetric constant matrix $M \in R^{n \times n}$, scalars $r_{1}, r_{2}$ satisfying $r_{1}<r_{2}$, a vector function $\omega:\left[r_{1}, r_{2}\right] \rightarrow R^{n}$ such that the integrations concerned are well defined, then

$$
\begin{gathered}
\left(\int_{r_{1}}^{r_{2}} \omega(s) d s\right)^{T} M\left(\int_{r_{1}}^{r_{2}} \omega(s) d s\right) \\
\quad \leq\left(r_{2}-r_{1}\right) \int_{r_{1}}^{r_{2}} \omega^{T}(s) M \omega(s) d s .
\end{gathered}
$$

Lemma 2 (see [29]). For given positive semidefinite matrices $Z_{1}$ and $Z_{2}$, if there exists a matrix $T$ such that

$$
\left[\begin{array}{cc}
Z_{1} & T \\
* & Z_{2}
\end{array}\right] \geq 0
$$

then the following inequality for any $\alpha(0<\alpha<1)$ holds

$$
\left[\begin{array}{cc}
\frac{1}{\alpha} Z_{1} & 0 \\
* & \frac{1}{1-\alpha} Z_{2}
\end{array}\right] \geq\left[\begin{array}{cc}
Z_{1} & T \\
* & Z_{2}
\end{array}\right]
$$

\section{Main Results}

3.1. Stability Analysis. Now, we give a new stability criterion for the origin of system (7) with (2) as follows.
Theorem 3. For given scalars $\tau_{i j}>0$ and $K_{i}(i \in \mathcal{N}, j \in \mathcal{S}(i))$, the origin of system (7) with (2) is globally asymptotically stable if there exist matrices $P_{i j}=P_{i j}^{T}>0, Q_{i j}=Q_{i j}^{T} \geq 0, R_{i j}=R_{i j}^{T} \geq$ $0, Z_{i j}=Z_{i j}^{T}>0$, and $U_{i j}=U_{i j}^{T}>0(\forall i \in \mathcal{N}, j \in \mathcal{S}(i))$ with appropriate dimensions, such that

$$
\begin{aligned}
& {\left[\begin{array}{cc}
Z_{i j} & T_{i j} \\
* & Z_{i j}
\end{array}\right] \geq 0,} \\
& {\left[\begin{array}{cc}
\Phi_{i j} & \Theta_{i j} \\
* & -U_{i j}
\end{array}\right]<0,}
\end{aligned}
$$

where $i=1,2, \ldots, n$ and $j \in \mathcal{S}(i)$, and

$$
\begin{aligned}
& \Phi_{i j}=\left[\begin{array}{cccc}
\Phi_{i j}^{11} & \frac{1}{\tau_{i j}}\left(Z_{i j}-T_{i j}\right) & \frac{1}{\tau_{i j}} T_{i j} & \Phi_{i j}^{14} \\
* & \Phi_{i j}^{22} & \frac{1}{\tau_{i j}}\left(Z_{i j}-T_{i j}\right) & 0 \\
* & * & -R_{i j}-\frac{1}{\tau_{i j}} Z_{i j} & 0 \\
* & * & * & \Phi_{i j}^{44}
\end{array}\right], \\
& \Phi_{i j}^{11}=\tau_{i j} Q_{i j}+R_{i j}-\frac{1}{\tau_{i j}} Z_{i j} \\
& -\frac{1}{m_{i}}\left(M_{i 1} B_{i} K_{i}+K_{i}^{T} B_{i}^{T} M_{i 1}^{T}-M_{i 1}\left(\sum_{l \in \mathscr{P}(i)} U_{l i}\right) M_{i 1}^{T}\right), \\
& \Phi_{i j}^{14}=P_{i j} \\
& +\frac{1}{m_{i}}\left(M_{i 1}-K_{i}^{T} B_{i}^{T} M_{i 2}^{T}+M_{i 1}\left(\sum_{l \in \mathscr{P}(i)} U_{l i}\right) M_{i 2}^{T}\right), \\
& \Phi_{i j}^{22}=-Q_{i j}-\frac{1}{\tau_{i j}}\left(2 Z_{i j}-T_{i j}-T_{i j}^{T}\right), \\
& \Phi_{i j}^{44}=P_{i j}+\tau_{i j} Z_{i j} \\
& +\frac{1}{m_{i}}\left(M_{i 2}+M_{i 2}^{T}+M_{i 2}\left(\sum_{l \in \mathscr{P}(i)} U_{l i}\right) M_{i 2}^{T}\right), \\
& \Theta_{i j}=\left[\begin{array}{llll}
0 & B_{d i j} K_{i} & 0 & 0
\end{array}\right]^{T} .
\end{aligned}
$$

Proof. The proof is provided in the Appendix.

Remark 4. In Theorem 3, a sufficient condition of globally asymptotical stability for the origin of system (7) with (2) is given in terms of LMIs. Different from the one in [20], positive definite matrices $P_{i j}, Q_{i j}, R_{i j}$, and $Z_{i j}$ correspond to the oriented link $(i, j)$, so the Lyapunov functional (A.1) is more general. Meanwhile, each inequality in (13) is also related to an oriented link $(i, j)$ in $\mathscr{L}$, so its dimension is lower. Combined with using a reciprocally convex technique [28], the computational complexity and the conservatism are reduced. 


\subsection{A Decentralized Routing Control Scheme}

3.2.1. Controller Design without the Physical Constraints. From (13), one can find that $M_{i 2}+M_{i 2}^{T}(i=1,2, \ldots, n)$ are negative definite, this implies that $M_{i 2}$ are nonsingular. Set

$$
\begin{gathered}
M_{i 1}=\varepsilon_{i} M_{i 2}, \quad \overline{M_{i 2}}=M_{i 2}^{-1}, \quad \bar{P}_{i j}=\overline{M_{i 2}} P_{i j}{\overline{M_{i 2}}}^{T}, \\
\bar{Q}_{i j}=\overline{M_{i 2}} Q_{i j}{\overline{M_{i 2}}}^{T}, \quad \bar{R}_{i j}=\bar{M}_{i 2} R_{i j}{\overline{M_{i 2}}}^{T}, \\
\bar{Z}_{i j}=\bar{M}_{i 2} Z_{i j}{\overline{M_{i 2}}}^{T}, \quad \bar{T}_{i j}={\overline{M_{i 2}}}_{i j}{\overline{M_{i 2}}}^{T}, \quad F_{i}=K_{i}{\overline{M_{i 2}}}^{T},
\end{gathered}
$$

pre- and postmultiplying the left side of inequality (13) by $\operatorname{diag}\left\{\bar{M}_{i 2}, \bar{M}_{i 2}, \bar{M}_{i 2}, \bar{M}_{i 2}, I\right\}$ and its transpose, and from the Schur complement, we get the following.

Theorem 5. For given scalars $\tau_{i j}>0$ and $\varepsilon_{i}(i \in \mathcal{N}, j \in \mathcal{S}(i))$, the origin of system (7) with (2) is globally asymptotically stable if there exist matrices $\bar{P}_{i j}=\bar{P}_{i j}^{T}>0, \bar{Q}_{i j}=\bar{Q}_{i j}^{T} \geq 0, \bar{R}_{i j}=\bar{R}_{i j}^{T} \geq$ 0 , and $\bar{Z}_{i j}=\bar{Z}_{i j}^{T}>0, F_{i}$ and $\bar{M}_{i 2}$ (for all $\left.i \in \mathcal{N}, j \in \mathcal{S}(i)\right)$ with appropriate dimensions, such that

$$
\begin{aligned}
& {\left[\begin{array}{cc}
\bar{Z}_{i j} & \bar{T}_{i j} \\
* & \bar{Z}_{i j}
\end{array}\right] \geq 0,} \\
& {\left[\begin{array}{cc}
\Omega_{i j} & \Gamma_{i j} \\
* & -U_{i j}
\end{array}\right]<0,}
\end{aligned}
$$

where $i \in \mathscr{N}$ and $j \in \mathcal{S}(i)$, and

$$
\begin{aligned}
& \Omega_{i j}=\left[\begin{array}{cccc}
\Omega_{i j}^{11} & \frac{1}{\tau_{i j}}\left(\bar{Z}_{i j}-\bar{T}_{i j}\right) & \frac{1}{\tau_{i j}} \bar{T}_{i j} & \Omega_{i j}^{14} \\
* & \Omega_{i j}^{22} & \frac{1}{\tau_{i j}}\left(\bar{Z}_{i j}-\bar{T}_{i j}\right) & 0 \\
* & * & -\bar{R}_{i j}-\frac{1}{\tau_{i j}} \bar{Z}_{i j} & 0 \\
* & * & * & \Omega_{i j}^{44}
\end{array}\right], \\
& \Gamma_{i j}=\left[\begin{array}{llll}
0 & B_{d i j} F_{i} & 0 & 0
\end{array}\right]^{T} \text {, } \\
& \Omega_{i j}^{11}=\tau_{i j} \bar{Q}_{i j}+\bar{R}_{i j}-\frac{1}{\tau_{i j}} \bar{Z}_{i j} \\
& -\frac{1}{m_{i}} \varepsilon_{i}\left(B_{i} F_{i}+F_{i}^{T} B_{i}^{T}-\varepsilon_{i} \sum_{l \in \mathscr{P}(i)} U_{l i}\right) \text {, } \\
& \Omega_{i j}^{14}=\bar{P}_{i j}+\frac{1}{m_{i}}\left(\bar{M}_{i 2}^{T}-F_{i}^{T} B_{i}^{T}+\sum_{l \in \mathscr{P}(i)} U_{l i}\right), \\
& \Omega_{i j}^{22}=-\bar{Q}_{i j}-\frac{1}{\tau_{i j}}\left(2 \bar{Z}_{i j}-\bar{T}_{i j}-\bar{T}_{i j}^{T}\right), \\
& \Omega_{i j}^{44}=\bar{P}_{i j}+\tau_{i j} \bar{Z}_{i j}+\frac{1}{m_{i}}\left(\bar{M}_{i 2}^{T}+\bar{M}_{i 2}+\sum_{l \in \mathscr{P}(i)} U_{l i}\right) .
\end{aligned}
$$

The gains $K_{i}(i \in \mathcal{N})$ of the subsystem controllers in (8) are given by

$$
K_{i}=F_{i} \bar{M}_{i 2}^{T}
$$

3.2.2. LMI-Based Conditions for the Physical Constraints. Now, we translate the physical constraints (3)-(5) into LMIbased conditions.

(1) Nonnegativity Constraint on Data Traffic Flow. From (1), it is easy to see that the nonnegativity of $x_{i}(k)$ and $f_{i j}^{d}(k)$ can be guaranteed if $I+B_{i} K_{i}$ and $K_{i}$ are nonnegative for all $i \in$ $\mathcal{N}, j \in \mathcal{S}(i)$, and $d \in \mathcal{N}_{d}^{i}$.

Note that the negative definiteness of $\bar{M}_{i 2}+\bar{M}_{i 2}^{T}$, and from (17), the nonnegativity of $I+B_{i} K_{i}$ and $K_{i}$ can be guaranteed if $\bar{M}_{i 2}$ are diagonal for all $i \in \mathcal{N}$ and

$$
\begin{gathered}
\bar{M}_{i 2}+B_{i} F_{i} \preceq 0, \\
F_{i} \preceq 0 .
\end{gathered}
$$

(2) Upper Bound on the Buffer Size. Note that

$$
\sum_{d \in \mathcal{N}_{d}^{i}} q_{i}^{d}(k)=H_{i} x_{i}(k),
$$

where

$$
H_{i}=[\underbrace{1}_{N_{d}^{i}} \begin{array}{lll}
1 & \cdots & 1
\end{array}] .
$$

So, the constraint (4) can be represented as

$$
0 \leq H_{i} x_{i}(k) \leq q_{i} .
$$

From $\Omega_{i j}<0$, it follows that $\Omega_{i j}^{44}<0$. So, there exist a small enough $\lambda>0$ such that

$$
\bar{P}_{i j}+\frac{1}{m_{i}}\left(\bar{M}_{i 2}^{T}+\bar{M}_{i 2}+\lambda I\right)<0 .
$$

Since

$$
-\frac{1}{\lambda} \bar{M}_{i 2} \bar{M}_{i 2}^{T} \leq \bar{M}_{i 2}^{T}+\bar{M}_{i 2}+\lambda I,
$$

and $\bar{P}_{i j}=\bar{M}_{i 2} P_{i j} \bar{M}_{i 2}^{T}$, inequality (24) implies

$$
\sum_{j \in \mathcal{S}(i)} P_{i j}<\frac{1}{\lambda} I .
$$

If (16) and (17) in Theorem 5 are feasible, under the assumption $x_{i}(\tau)=0$ for all $\tau \in\left\{-1,-2, \ldots,-\tau_{i j}\right\}$, from

$$
\begin{aligned}
x_{i}^{T}(k) & \left(\sum_{j \in \mathcal{S}(i)} P_{i j}\right) x_{i}(k) \\
& \leq \sum_{i=1}^{N} x_{i}^{T}(k)\left(\sum_{j \in \mathcal{S}(i)} P_{i j}\right) x_{i}(k) \leq V(k),
\end{aligned}
$$




$$
\begin{aligned}
V(0) & \leq \frac{1}{\lambda} \sum_{i=1}^{N} x_{i}^{T}(0) x_{i}(0) \\
& \leq \frac{1}{\lambda} \sum_{i=1}^{N} x_{i}^{T}(0) H_{i}^{T} H_{i} x_{i}(0) \leq \frac{1}{\lambda} \sum_{i=1}^{N} q_{i}^{2},
\end{aligned}
$$

we get

$$
x_{i}^{T}(k)\left(\sum_{j \in \mathcal{S}(i)} P_{i j}\right) x_{i}(k) \leq \frac{1}{\lambda} \sum_{i=1}^{N} q_{i}^{2},
$$

where $V(k)$ is defined in (A.1).

Thus, (23) can be guaranteed if

$$
\frac{1}{m_{i} q_{i}^{2}} H_{i}^{T} H_{i} \leq \frac{\lambda}{\sum_{i=1}^{N} q_{i}^{2}} P_{i j}, \quad \forall j \in \mathcal{S}(i) .
$$

From the Schur complement, the above inequality is equivalent to

$$
\left[\begin{array}{cc}
-\bar{P}_{i j} & \bar{M}_{i 2} H_{i}^{T} \\
* & -\frac{m_{i} q_{i}^{2}}{\sum_{i=1}^{N} q_{i}^{2}} \lambda
\end{array}\right]<0 .
$$

So, from the above analysis, it is known that (23) can be guaranteed if (24) and (30) hold for any $(i, j) \in \mathscr{L}$.

(3) Capacity Constraints on Links. For $j \in \mathcal{S}(i)$, it is known that there exists a nonnegative integer $i_{j}$ such that the numbers of $i$ nodes contained in $\delta(i)$ are less than $j$ and the ones of $m_{i}-i_{j}-1$ nodes contained in $\mathcal{S}(i)$ are greater than $j$. Thus, We get

$$
\sum_{d \in \mathcal{N}_{d}^{i}} f_{i j}^{d}(k)=G_{i j} u_{i}(k), \quad \forall(i, j) \in \mathscr{L},
$$

where

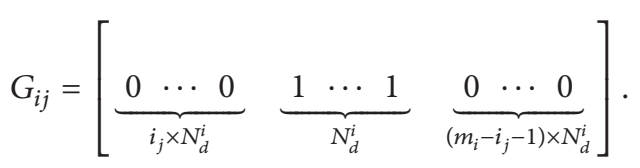

so the constraint (5) can be represented as

$$
0 \leq G_{i j} u_{i}(k) \leq c_{i j}
$$

Similarly, constraint (33) can be satisfied if (24) and

$$
\left[\begin{array}{cc}
-\bar{P}_{i j} & F_{i}^{T} G_{i j}^{T} \\
* & -\frac{c_{i j}^{2}}{\sum_{i=1}^{N} q_{i}^{2}} \lambda
\end{array}\right]<0 .
$$

Therefore, considering the physical constraints (3)-(5), a decentralized routing control scheme can be get as follows.

Theorem 6. For given scalars $\tau_{i j}>0$ and $\varepsilon_{i}(i \in \mathcal{N}, j \in \mathcal{S}(i))$, the origin of system (7) with (2) is globally asymptotically stable

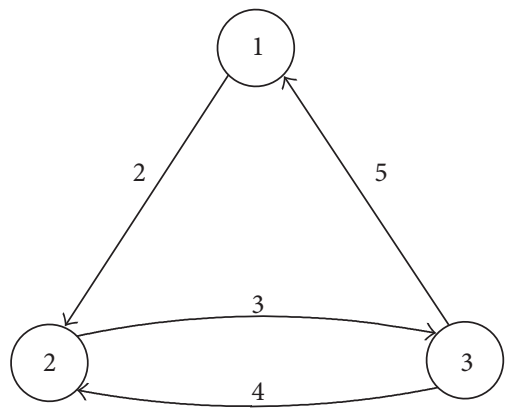

FIGURE 1: Topology of a communication network.

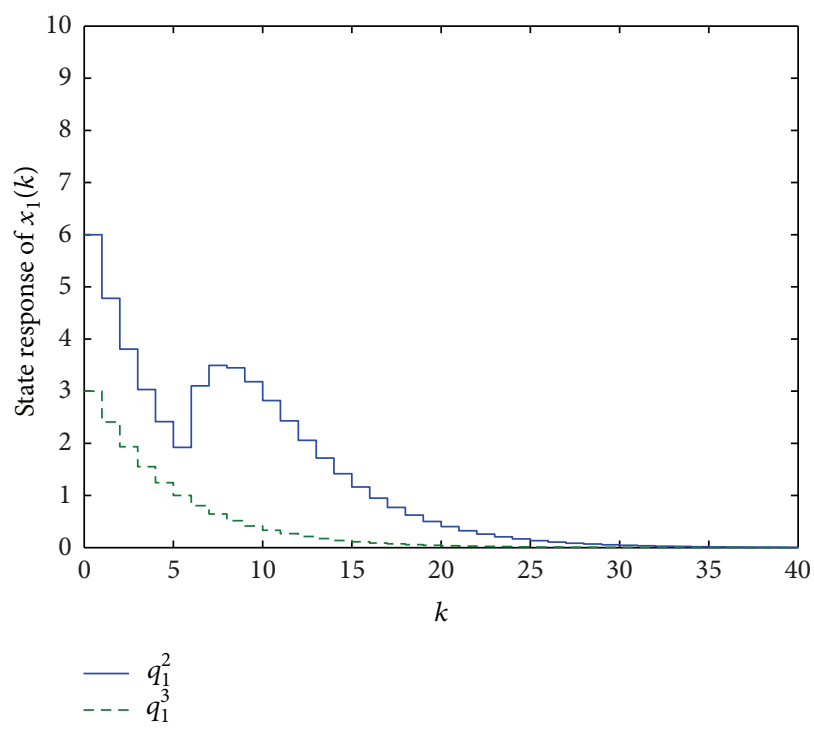

FIGURE 2: State response of $x_{1}(k)$.

if there exist a scalar $\lambda>0$, matrices $\bar{P}_{i j}=\bar{P}_{i j}^{T}>0, \bar{Q}_{i j}=\bar{Q}_{i j}^{T} \geq$ $0, \bar{R}_{i j}=\bar{R}_{i j}^{T} \geq 0$, and $\bar{Z}_{i j}=\bar{Z}_{i j}^{T}>0, F_{i}$ and diagonal matrices $\bar{M}_{i 2}$ (for all $i \in \mathcal{N}, j \in \mathcal{S}(i)$ ) with appropriate dimensions, such that (16), (17), (20), (24), (30), and (34) hold.

Correspondingly, the gains $K_{i}$ of the subsystem controllers in (8) are given by (19).

Remark 7. In $[20,25]$, a scalar $\lambda$ was needed to be selected before and the restriction $V(0)=0$ in transforming the physical constraints into LMI-based conditions. Contrastively, $\lambda$ involved in inequalities (30) and (34) is a variable and it can be found by solving these LMIs. More importantly, the restriction $V(0)=0$ is not needed here.

Remark 8. Different from [20, 25], positive definite matrix $\bar{P}$ in Theorem 6 is not required to be diagonal, which discards the constraint on $P$.

\section{Numerical Example}

Consider a data communication network shown in Figure 1 $[20,30]$. The capacity of each link is also indicated in 
the figure, the unit of which is $\mathrm{kbit} / \mathrm{sec}$. All the nodes are assumed to be sources as well as destinations. From Figure 1, it follows that there are 6 queues (the states of the system) and 8 output flows for these queues (the input signals). The queue dynamic of the communication network can be described as follows

$$
\begin{aligned}
x_{1}(k+1)= & x_{1}(k)+B_{1} u_{1}(k)+B_{d 31} u_{3}\left(k-\tau_{31}(k)\right), \\
x_{2}(k+1)= & x_{2}(k)+B_{2} u_{2}(k)+B_{d 12} u_{1}\left(k-\tau_{12}(k)\right) \\
& +B_{d 32} u_{3}\left(k-\tau_{32}(k)\right), \\
x_{3}(k+1)= & x_{3}(k)+B_{3} u_{3}(k)+B_{d 23} u_{2}\left(k-\tau_{23}(k)\right),
\end{aligned}
$$

where

$$
\begin{aligned}
& x_{1}(k)=\left[\begin{array}{c}
q_{1}^{2}(k) \\
q_{1}^{3}(k)
\end{array}\right], \quad x_{2}(k)=\left[\begin{array}{l}
q_{2}^{1}(k) \\
q_{2}^{3}(k)
\end{array}\right] \text {, } \\
& x_{3}(k)=\left[\begin{array}{c}
q_{3}^{1}(k) \\
q_{3}^{2}(k)
\end{array}\right], \quad u_{1}(k)=\left[\begin{array}{c}
f_{12}^{2}(k) \\
f_{12}^{3}(k)
\end{array}\right] \text {, } \\
& u_{2}(k)=\left[\begin{array}{c}
f_{23}^{1}(k) \\
f_{23}^{3}(k)
\end{array}\right], \quad u_{3}(k)=\left[\begin{array}{c}
f_{31}^{1}(k) \\
f_{31}^{2}(k) \\
f_{32}^{1}(k) \\
f_{32}^{2}(k)
\end{array}\right] \text {, } \\
& B_{1}=B_{2}=\left[\begin{array}{cc}
-1 & 0 \\
0 & -1
\end{array}\right], \quad B_{d 31}=\left[\begin{array}{llll}
0 & 1 & 0 & 0 \\
0 & 0 & 0 & 0
\end{array}\right] \text {, } \\
& B_{d 12}=\left[\begin{array}{ll}
0 & 0 \\
0 & 1
\end{array}\right], \quad B_{d 32}=\left[\begin{array}{llll}
0 & 0 & 1 & 0 \\
0 & 0 & 0 & 0
\end{array}\right] \text {, } \\
& B_{3}=\left[\begin{array}{cccc}
-1 & 0 & -1 & 0 \\
0 & -1 & 0 & -1
\end{array}\right], \quad B_{d 23}=\left[\begin{array}{ll}
1 & 0 \\
0 & 0
\end{array}\right] \text {. }
\end{aligned}
$$

Assume that the maximal buffer sizes, $q_{i}(i \in \mathcal{N})$, are given by $q_{1}=10, q_{2}=15$, and $q_{3}=20$. For the case of $\tau_{i j}=5$ for all $(i, j) \in \mathscr{L}$, by Theorem 6 with $\varepsilon_{i}=4$, one can get

$$
\begin{gathered}
K_{1}=\left[\begin{array}{ll}
0.2035 & 0.0001 \\
0.0001 & 0.1971
\end{array}\right], \quad K_{2}=\left[\begin{array}{cc}
0.1934 & 0 \\
0 & 0.2037
\end{array}\right], \\
K_{3}=\left[\begin{array}{cc}
0.2219 & 0 \\
0 & 0.1743 \\
0.1388 & 0 \\
0 & 0.1736
\end{array}\right] .
\end{gathered}
$$

Now, we provide a simulation.

Suppose that the initial states of $x_{i}(k)$ are given by $x_{1}(0)=$ $[6,3]^{T}, x_{2}(0)=[5,8]^{T}$ and $x_{3}(0)=[9,9]^{T}$. With the obtained controller gains in (37), the state responses of the system are depicted in Figures 2, 3, and 4, which shows that the system is asymptotically stable. Figures $5,6,7$, and 8 show

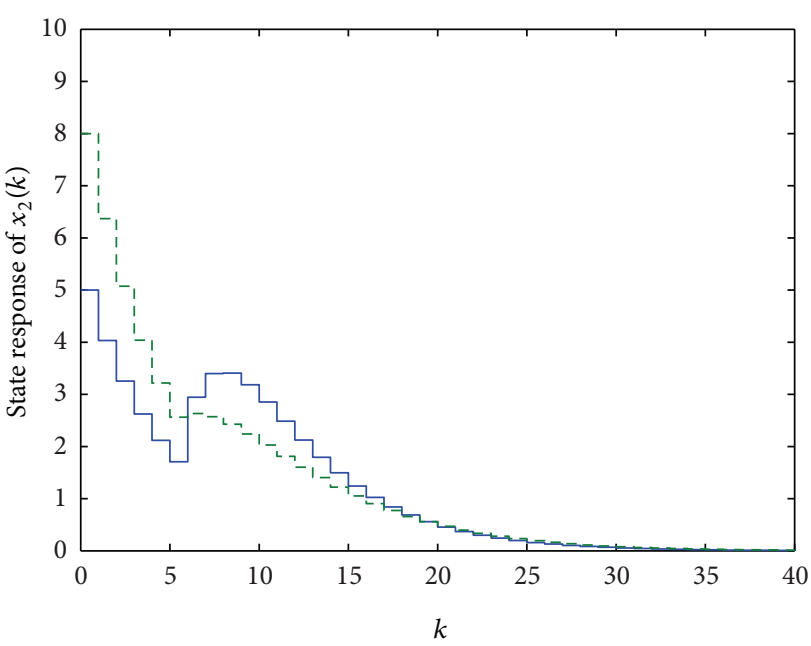

$\begin{aligned}- & q_{2}^{1} \\ --- & q_{2}^{3}\end{aligned}$

FIGURE 3: State response of $x_{2}(k)$.

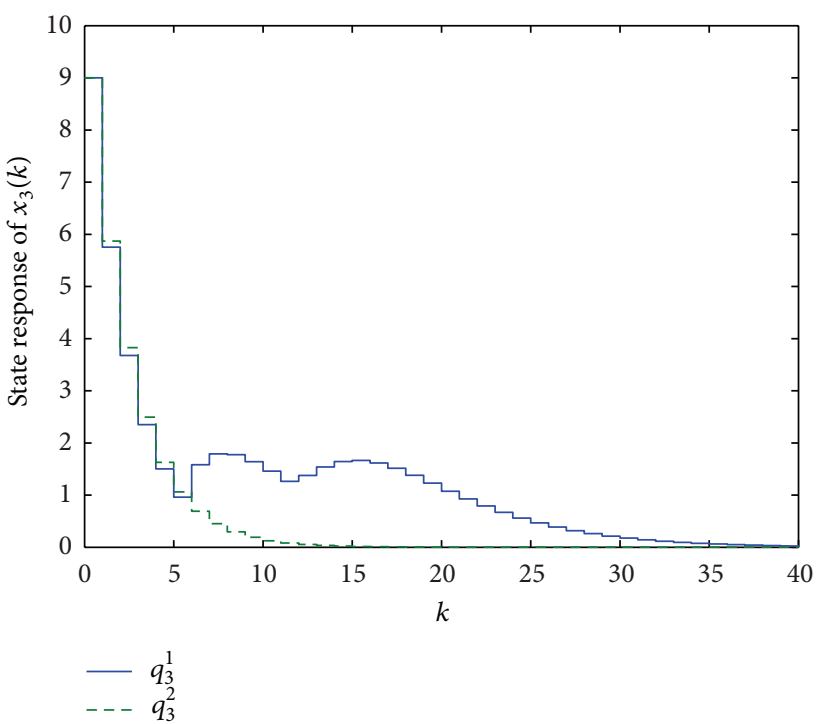

FIGURE 4: State response of $x_{3}(k)$.

the data flows between nodes in the communication network. From Figures 5-8, one can see that the physical constraints are satisfied although initial state $x(0)$ is not equal to 0 . So, the proposed decentralized routing control scheme is effective.

\section{Conclusion}

In this paper, the routing control problem of data communication networks has been investigated. By considering both the network-induced delays and the physical constraints, a decentralized routing control scheme is proposed, which can be realized by solving a set of LMIs. Since a method is used to relax the transformation conditions of the physical constraints, and a reciprocally convex combination technique is 


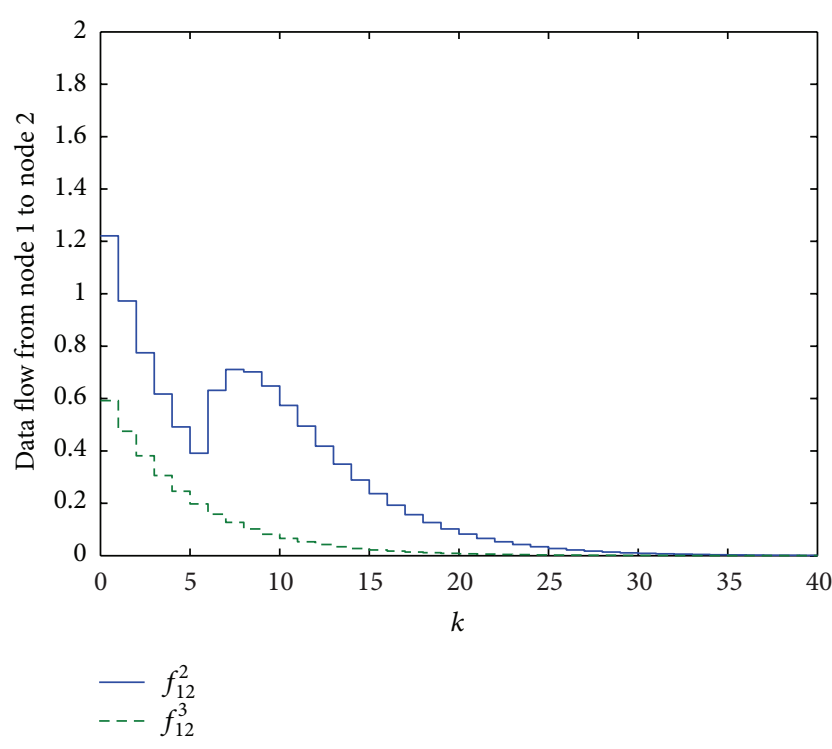

FIGURE 5: Data flow from node 1 to node 2.

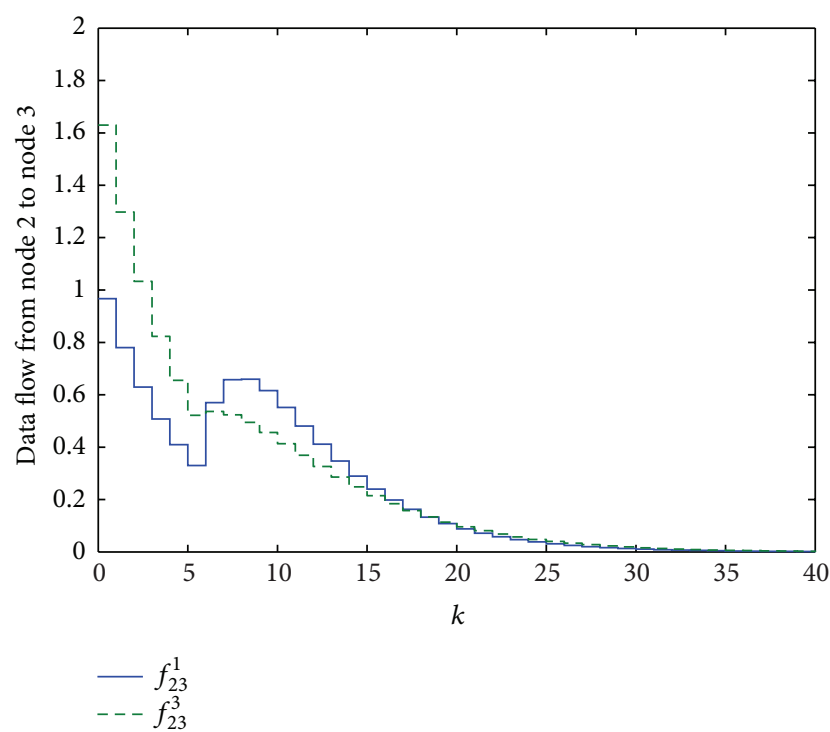

Figure 6: Data flow from node 2 to node 3.

employed to dealing with the network-induced delays, a less complex and less conservative designing result is obtained. A simulation example is given to illustrate the effectiveness of the presented designing scheme.

\section{Appendix}

Proof of Theorem 3. Choose a Lyapunov functional as

$$
V(k)=V_{1}(k)+V_{2}(k)+V_{3}(k)+V_{4}(k),
$$

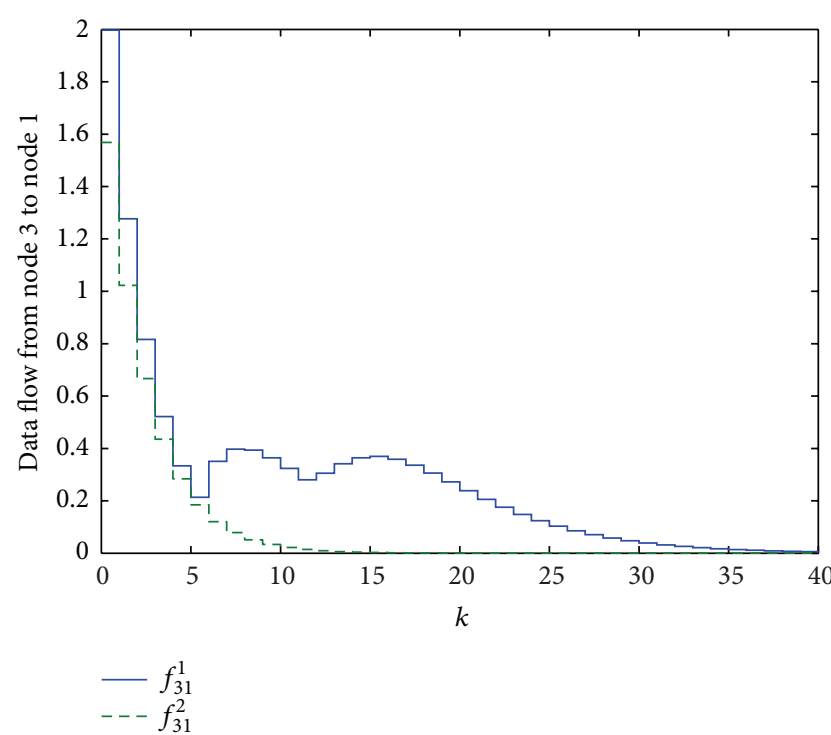

Figure 7: Data flow from node 3 to node 1.

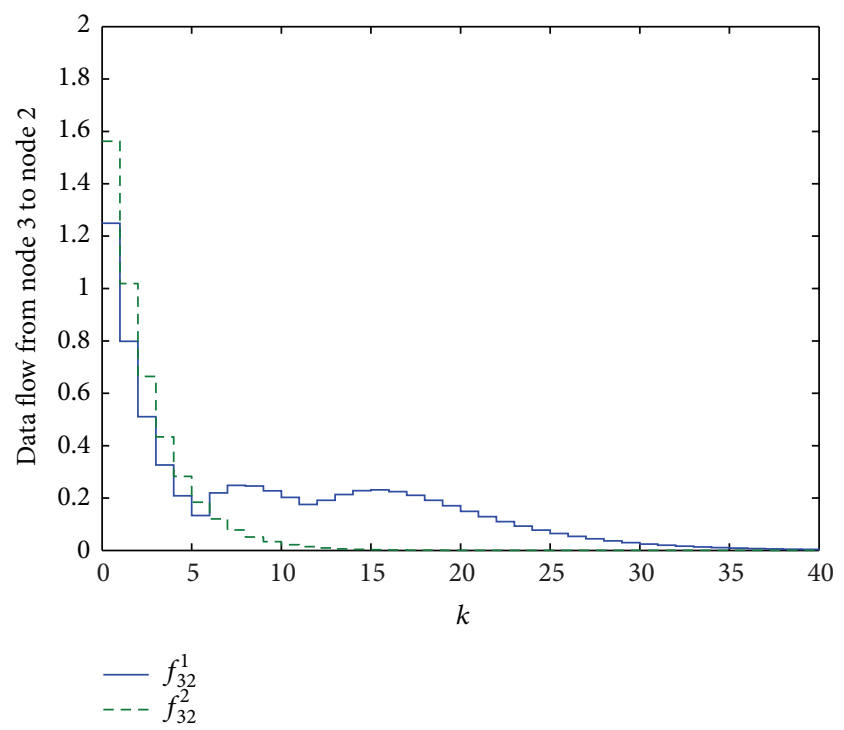

FIGURE 8: Data flow from node 3 to node 2.

where

$$
\begin{aligned}
V_{1}(k)=\sum_{i=1}^{n} \sum_{j \in \mathscr{P}(i)} x_{j}^{T}(k) P_{j i} x_{j}(k), \\
V_{2}(k)=\sum_{i=1}^{n} \sum_{j \in \mathscr{P}(i)}\left(\sum_{l=k-\tau_{j i}(k)}^{k-1} x_{j}^{T}(l) Q_{j i} x_{j}(l)\right. \\
\\
\left.\quad+\sum_{l=-\tau_{j i}+1}^{0} \sum_{r=k+l}^{k-1} x_{j}^{T}(r) Q_{j i} x_{j}(r)\right),
\end{aligned}
$$




$$
\begin{aligned}
V_{3}(k) & =\sum_{i=1}^{n} \sum_{j \in \mathscr{P}(i)} \sum_{l=k-\tau_{j i}}^{k-1} x_{j}^{T}(l) R_{j i} x_{j}(l), \\
V_{4}(k) & =\sum_{i=1}^{n} \sum_{j \in \mathscr{P}(i)} \sum_{l=-\tau_{j i}}^{-1} \sum_{r=k+l}^{k-1} \eta_{j}^{T}(r) Z_{j i} \eta_{j}(r),
\end{aligned}
$$$$
=\sum_{i=1}^{n} \sum_{j \in \mathscr{P}(i)}\left(\tau_{j i} x_{j}^{T}(k) Q_{j i} x_{j}(k)\right.
$$

$$
\left.-x_{j}^{T}\left(k-\tau_{j i}(k)\right) Q_{j i} x_{j}\left(k-\tau_{j i}(k)\right)\right)
$$$$
=\sum_{i=1}^{n} \sum_{j \in \mathcal{S}(i)}\left(\tau_{i j} x_{i}^{T}(k) Q_{i j} x_{i}(k)\right.
$$$$
\left.-x_{i}^{T}\left(k-\tau_{i j}(k)\right) Q_{i j} x_{i}\left(k-\tau_{i j}(k)\right)\right),
$$

and $P_{i j}=P_{i j}^{T}>0, Q_{i j}=Q_{i j}^{T} \geq 0, R_{i j}=R_{i j}^{T} \geq 0, Z_{i j}=Z_{i j}^{T}>$ 0 (for all $\in \mathcal{N}, j \in \mathcal{S}(i)$ ) are to be determined.

Note that

$$
\sum_{i=1}^{n} \sum_{j \in \mathscr{P}(i)} x_{j}^{T}(k) P_{j i} x_{j}(k)=\sum_{i=1}^{n} \sum_{j \in \mathcal{S}(i)} x_{i}^{T}(k) P_{i j} x_{i}(k),
$$

We get

$$
\begin{aligned}
& \Delta V_{1}(k) \\
& =\sum_{i=1}^{n} \sum_{j \in \mathcal{S}(i)}\left(x_{i}^{T}(k+1) P_{i j} x_{i}(k+1)-x_{i}^{T}(k) P_{i j} x_{i}^{T}(k)\right) \\
& =\sum_{i=1}^{n} \sum_{j \in \mathcal{S}(i)}\left(\eta_{i}^{T}(k) P_{i j} \eta_{i}(k)+\eta_{i}^{T}(k) P_{i j} x_{i}(k)\right. \\
& \left.+x_{i}^{T}(k) P_{i j} \eta_{i}^{T}(k)\right) .
\end{aligned}
$$

$\Delta V_{3}(k)$

$$
\begin{aligned}
& =\sum_{i=1}^{n} \sum_{j \in \mathscr{P}(i)}\left(x_{j}^{T}(k) R_{j i} x_{j}(k)-x_{j}^{T}\left(k-\tau_{j i}\right) R_{j i} x_{j}\left(k-\tau_{j i}\right)\right) \\
& =\sum_{i=1}^{n} \sum_{j \in \mathcal{S}(i)}\left(x_{i}^{T}(k) R_{i j} x_{i}(k)-x_{i}^{T}\left(k-\tau_{i j}\right) R_{i j} x_{i}\left(k-\tau_{i j}\right)\right),
\end{aligned}
$$

$\Delta V_{4}(k)$

$$
\begin{aligned}
& =\sum_{i=1}^{n} \sum_{j \in \mathscr{P}(i)}\left(\tau_{j i} \eta_{j}^{T}(k) Z_{j i} \eta_{j}(k)-\sum_{l=k-\tau_{j i}}^{k-1} \eta_{j}^{T}(l) Z_{j i} \eta_{j}(l)\right) \\
& =\sum_{i=1}^{n} \sum_{j \in \mathcal{S}(i)}\left(\tau_{i j} \eta_{i}^{T}(k) Z_{i j} \eta_{i}(k)-\sum_{l=k-\tau_{i j}}^{k-1} \eta_{i}^{T}(l) Z_{i j} \eta_{i}(l)\right) .
\end{aligned}
$$

Similarly, taking the differences of $V_{i}(k)(i=2,3,4)$ along the trajectory of (7) yields that

Since

$$
\begin{gathered}
\Delta V_{2}(k) \\
=\sum_{i=1}^{n} \sum_{j \in \mathscr{P}(i)}\left\{\left(\sum_{l=k+1-\tau_{j i}(k+1)}^{k} x_{j}^{T}(l) Q_{j i} x_{j}(l)\right.\right. \\
\left.\quad-\sum_{l=k-\tau_{j i}(k)}^{k-1} x_{j}^{T}(l) Q_{j i} x_{j}(l)\right) \\
+\sum_{l=-\tau_{j i}+1}^{0}\left(x_{j}^{T}(k) Q_{j i} x_{j}(k)\right. \\
\left.\left.-x_{j}^{T}(k+l) Q_{j i} x_{j}(k+l)\right)\right\} \\
\left.\begin{array}{l}
\sum_{i=1}^{n} \sum_{j \in \mathscr{P}(i)} \sum_{l=k+1-\tau_{j i}} x_{j}^{T}(l) Q_{j i} x_{j}(l) \\
-x_{j}^{T}\left(k-\tau_{i j}(k)\right) Q_{j i} x_{j}\left(k-\tau_{i j}(k)\right) \\
+\tau_{j i} x_{j}^{T}(k) Q_{j i} x_{j}(k) \\
-\sum_{l=k-\tau_{j i}+1}^{k} x_{j}^{T}(l) Q_{j i} x_{j}(l)
\end{array}\right)
\end{gathered}
$$$$
\begin{aligned}
& -\sum_{l=k-\tau_{i j}}^{k-1} \eta_{i}^{T}(l) Z_{i j} \eta_{i}(l) \\
& =-\sum_{l=k-\tau_{i j}(k)}^{k-1} \eta_{i}^{T}(l) Z_{i j} \eta_{i}(l)-\sum_{l=k-\tau_{i j}}^{k-\tau_{i j}(k)-1} \eta_{i}^{T}(l) Z_{i j} \eta_{i}(l),
\end{aligned}
$$

and from Lemma 1, we get

$$
\begin{aligned}
& -\sum_{l=k-\tau_{i j(k)}}^{k-1} \eta_{i}^{T}(l) Z_{i j} \eta_{i}(l) \\
& \leq-\frac{1}{\tau_{i j}(k)}\left[x_{i}^{T}(k)-x_{i}^{T}\left(k-\tau_{i j}(k)\right)\right] \\
& \quad \times Z_{i j}\left[x_{i}(k)-x_{i}\left(k-\tau_{i j}(k)\right)\right], \\
& -\sum_{l=k-\tau_{i j}}^{k-\tau_{i j}(k)-1} \eta_{i}^{T}(l) Z_{i j} \eta_{i}(l) \\
& \leq-\frac{1}{\tau_{i j}-\tau_{i j}(k)}\left[x_{i}^{T}\left(k-\tau_{i j}(k)\right)-x_{i}^{T}\left(k-\tau_{i j}\right)\right] Z_{i j} \\
& \times\left[x_{i}\left(k-\tau_{i j}(k)\right)-x_{i}\left(k-\tau_{i j}\right)\right] .
\end{aligned}
$$


Thus, from Lemma 2, it yields that

$$
\begin{aligned}
& -\sum_{l=k-\tau_{i j}}^{k-1} \eta_{i}^{T}(l) Z_{i j} \eta_{i}(l) \\
& \left.\leq-\frac{1}{\tau_{i j}}\left[\begin{array}{c}
x_{i}(k)-x_{i}\left(k-\tau_{i j}(k)\right) \\
x_{i}\left(k-\tau_{i j}(k)\right)-x_{i}\left(k-\tau_{i j}\right)
\end{array}\right]\right]^{T}\left[\begin{array}{cc}
Z_{i j} & T_{i j} \\
* & Z_{i j}
\end{array}\right] \\
& \quad \times\left[\begin{array}{c}
x_{i}(k)-x_{i}\left(k-\tau_{i j}(k)\right) \\
x_{i}\left(k-\tau_{i j}(k)\right)-x_{i}\left(k-\tau_{i j}\right)
\end{array}\right] .
\end{aligned}
$$

On the other hand, for any matrices $M_{i 1}, M_{i 2}$, and $U_{i j}=$ $U_{i j}^{T}>0$ with appropriate dimensions, it follows that

$$
\begin{aligned}
& 0=2 \sum_{i=1}^{n}\left[x_{i}^{T}(k) M_{i 1}+\eta_{i}^{T}(k) M_{i 2}\right] \\
& \times\left[\eta_{i}(k)-B_{i} K_{i} x_{i}(k)-\sum_{j \in \mathscr{P}(i)} B_{d j i} K_{j} x_{j}\left(k-\tau_{j i}(k)\right)\right] \\
& \leq 2 \sum_{i=1}^{n}\left[x_{i}^{T}(k) M_{i 1}+\eta_{i}^{T}(k) M_{i 2}\right]\left[\eta_{i}(k)-B_{i} K_{i} x_{i}(k)\right] \\
& +\sum_{i=1}^{n} \sum_{j \in \mathscr{P}(i)}\left(\left[x_{i}^{T}(k) M_{i 1}+\eta_{i}^{T}(k) M_{i 2}\right]\right. \\
& \times U_{j i}\left[x_{i}^{T}(k) M_{i 1}+\eta_{i}^{T}(k) M_{i 2}\right]^{T} \\
& +x_{j}^{T}\left(k-\tau_{j i}(k)\right) K_{j}^{T} B_{d j i}^{T} \\
& \left.\times U_{j i}^{-1} B_{d j i} K_{j} x_{j}\left(k-\tau_{j i}(k)\right)\right) \\
& =2 \sum_{i=1}^{n}\left[x_{i}^{T}(k) M_{i 1}+\eta_{i}^{T}(k) M_{i 2}\right]\left[\eta_{i}(k)-B_{i} K_{i} x_{i}(k)\right] \\
& +\sum_{i=1}^{n}\left(\left[x_{i}^{T}(k) M_{i 1}+\eta_{i}^{T}(k) M_{i 2}\right]\right. \\
& \times\left(\sum_{l \in \mathscr{P}(i)} U_{l i}\right)\left[x_{i}^{T}(k) M_{i 1}+\eta_{i}^{T}(k) M_{i 2}\right]^{T} \\
& +\sum_{l \in \mathscr{P}(i)} x_{j}^{T}\left(k-\tau_{j i}(k)\right) \\
& \left.\times K_{j}^{T} B_{d j i}^{T} U_{j i}^{-1} B_{d j \mathrm{i}} K_{j} x_{j}\left(k-\tau_{j i}(k)\right)\right)
\end{aligned}
$$

$$
\begin{aligned}
=\sum_{i=1}^{n} \sum_{j \in \mathcal{S}(i)}\left\{\frac{1}{m_{i}}\right. & \\
\times & \left(2\left[x_{i}^{T}(k) M_{i 1}+\eta_{i}^{T}(k) M_{i 2}\right]\right. \\
& \times\left[\eta_{i}(k)-B_{i} K_{i} x_{i}(k)\right] \\
& +\left[x_{i}^{T}(k) M_{i 1}+\eta_{i}^{T}(k) M_{i 2}\right]\left(\sum_{l \in \mathscr{P}(i)} U_{l i}\right) \\
& \left.\times\left[x_{i}^{T}(k) M_{i 1}+\eta_{i}^{T}(k) M_{i 2}\right]^{T}\right) \\
+ & x_{i}^{T}\left(k-\tau_{i j}(k)\right) K_{i}^{T} B_{d i j}^{T} U_{i j}^{-1} \\
& \left.\times B_{d i j} K_{i} x_{i}\left(k-\tau_{i j}(k)\right)\right\} .
\end{aligned}
$$

So, combining (A.4)-(A.9), we get that

$$
\Delta V(k) \leq \sum_{i=1}^{n} \sum_{j \in \mathcal{S}(i)} \zeta_{i j}^{T}(k) \Psi_{i j} \zeta_{i j}(k)
$$

where

$$
\begin{gathered}
\zeta_{i j}(k)=\left[\begin{array}{cccc}
x_{i}^{T}(k) & x_{i}^{T}\left(k-\tau_{i j}(k)\right) & x_{i}^{T}\left(k-\tau_{i j}\right) & \eta_{i}^{T}(k)
\end{array}\right]^{T}, \\
\Psi_{i j}=\left[\begin{array}{cccc}
\Phi_{i j}^{11} & \frac{1}{\tau_{i j}}\left(Z_{i j}-T_{i j}\right) & \frac{1}{\tau_{i j}} T_{i j} & \Phi_{i j}^{14} \\
* & \Psi_{i j}^{22} & \frac{1}{\tau_{i j}}\left(Z_{i j}-T_{i j}\right) & 0 \\
* & * & -R_{i j}-\frac{1}{\tau_{i j}} Z_{i j} & 0 \\
* & * & * & \Phi_{i j}^{44}
\end{array}\right], \\
\Psi_{i j}^{22}=\Phi_{i j}^{22}+K_{i}^{T} B_{d i j}^{T} U_{i j}^{-1} B_{d i j} K_{i} .
\end{gathered}
$$

From the Schur complement, it is known that the globally asymptotical stability of system (7) can be guaranteed if (12) and (13) hold for any $i \in \mathcal{N}$ and $j \in \mathcal{S}(i)$.

Thus, the proof is completed.

\section{Acknowledgments}

This work was supported by the National High Technology Research and Development Programme of China (863 Programme) under Grant 2009AA012201, the National Natural Science Foundation of China under Grant 61174085, the Training Excellent Young Teachers in Shanghai Universities (B37-0109-10-008), the Shanghai University Innovation Foundation (Grant no. A.10-0109-10-014), and the IndustryUniversity-Research Project of Shanghai. 


\section{References}

[1] L. Chen, S. H. Low, M. Chiangs, and J. C. Doyle, "Cross-layer congestion control, routing and scheduling design in ad hoc wireless networks," in Proceedings of the 25th IEEE International Conference on Computer Communications (INFOCOM '06), pp. 1-13, April 2006.

[2] D. Berteskas and R. Gallager, Data Networks, Prentice Hall, 2nd edition, 1992.

[3] L. R. W. Tymes, "Routing and flow control in TYMNET", IEEE Transactions on Communications Systems, vol. 29, no. 4, pp. 392398, 1981.

[4] J. H. Chang and L. Tassiulas, "Maximum lifetime routing in wireless sensor networks," IEEE/ACM Transactions on Networking, vol. 12, no. 4, pp. 609-619, 2004.

[5] T. P. Vum, "The design and analysis of a semi-dynamic routing rule," IEEE Transactions on Communications, vol. 29, no. 4, pp. 495-504, 1981.

[6] D. A. Tran and H. Raghavendra, "Congestion adaptive routing in mobile ad hoc networks," IEEE Transactions on Parallel and Distributed Systems, vol. 17, no. 11, pp. 1294-1305, 2006.

[7] E. J. Anderson and T. E. Anderson, "On the stability of adaptive routing in the presence of congestion control," in Proccedings of 23rd IEEE International Conference on Computer Communications (INFOCOM '03), pp. 948-958, April 2003.

[8] F. Kelly and Th. Voice, "Stability of end-to-end algorithms for joint routing and rate control," ACM SIGCOMM Computer Communication Review, vol. 35, no. 2, pp. 5-12, 2005.

[9] K. Akkaya and M. Younis, "Energy-aware routing to a mobile gateway in wireless sensor networks," in Proceedings of the IEEE Global Telecommunications Conference Workshops (GLOBECOM '04), pp. 16-21, December 2004.

[10] J. Zhang, Q. Zhang, B. Li, X. Luo, and W. Zhu, "Energy-efficient routing in mobile ad hoc networks: mobility-assisted case," IEEE Transactions on Vehicular Technology, vol. 55, no. 1, pp. 369-379, 2006.

[11] M. J. Neely, E. Modiano, and C. E. Rohrs, "Dynamic power allocation and routing for time-varying wireless networks," IEEE Journal on Selected Areas in Communications, vol. 23, no. 1, pp. 89-103, 2005.

[12] N. A. Kuznetsov and V. N. Fetisov, "Robust control of information networks," Automation and Remote Control, vol. 66, no. 9, pp. 1440-1453, 2005.

[13] A. Segall, "The modeling of adaptive routing in data-communication networks," IEEE Transactions on Communications, vol. 25, no. 1, pp. 85-95, 1977.

[14] F. H. Moss and A. Segall, "An optimal control approach to dynamic routing in networks," IEEE Transactions on Automatic Control, vol. 27, no. 2, pp. 329-339, 1982.

[15] D. Oulai, S. Chamberland, and S. Pierre, "A new routing-based admission control for MPLS networks," IEEE Communications Letters, vol. 11, no. 2, pp. 216-218, 2007.

[16] Y. Xi and E. M. Yeh, "Optimal capacity allocation, routing and congestion control in wireless networks," in Proceedings of the IEEE International Symposium on Information Theory (ISIT '06), pp. 2511-2515, July 2006.

[17] P. K. Pothuri, V. Sarangan, and J. P. Thomas, "Delay-constrained, energy-efficient routing in wireless sensor networks through topology control," in Proceedings of the IEEE International Conference on Networking, Sensing and Control (ICNSC '06), pp. 35-41, April 2006.
[18] A. Iftar and E. J. Davison, "Decentralized control strategies for dynamic routing," Optimal Control Applications \& Methods, vol. 23, no. 6, pp. 329-355, 2002.

[19] M. Baglietto, T. Parisini, and R. Zoppoli, "Distributedinformation neural control: the case of dynamic routing in trafic networks," IEEE Transactions on Neural Networks, vol. 12, no. 3, pp. 485-502, 2001.

[20] F. Abdollahi and K. Khorasani, "A novel $H_{\infty}$ control strategy for design of a robust dynamic routing algorithm in traffic networks," IEEE Journal on Selected Areas in Communications, vol. 26, no. 4, pp. 706-718, 2008.

[21] Y. Tang, H. Gao, W. Zou, and J. Kurths, "Distributed synchronization in networks of agent systems with nonlinearities and random switchings," IEEE Transactions on Cybernetics, , vol. 43, no. 1, pp. 358-370, 2013.

[22] Y. Tang, Z. Wang, H. Gao, S. Swift, and J. Kurths, "A constrained evolutionary computation method for detecting controlling regions of cortical networks," IEEE/ACM Transactions on Computational Biology and Bioinformatics, vol. 9, no. 6, pp. 15691581, 2012.

[23] D. Yue, E. Tian, Z. Wang, and J. Lam, "Stabilization of systems with probabilistic interval input delays and its applications to networked control systems," IEEE Transactions on Systems, Man and Cybernetics A, vol. 39, no. 4, pp. 939-945, 2009.

[24] E. Tian, D. Yue, T. C. Yang, Z. Gu, and G. Lu, "T-S fuzzy modelbased robust stabilization for networked control systems with probabilistic sensor and actuator failure," IEEE Transactions on Fuzzy Systems, vol. 19, no. 3, pp. 553-561, 2011.

[25] F. Abdollahi and K. Khorasani, "A decentralized Markovian jump $H_{\infty}$ control routing strategy for mobile multi-agent networked systems," IEEE Transactions on Control Systems Technology, vol. 19, no. 2, pp. 269-283, 2011.

[26] P. Naghshtabrizi, J. P. Hespanha, and A. R. Teel, "Exponential stability of impulsive systems with application to uncertain sampled-data systems," Systems \& Control Letters, vol. 57, no. 5, pp. 378-385, 2008.

[27] K. Gu, "An integral inequality in the stability problem of timedelay systems," in Proceedings of the 39th IEEE Conference on Decision and Control, pp. 2805-2810, 2000.

[28] P. Park, J. W. Ko, and C. Jeong, "Reciprocally convex approach to stability of systems with time-varying delays," Automatica, vol. 47, no. 1, pp. 235-238, 2011.

[29] X. Zhu and Y. Wang, "Parameter-dependent switching law for linear switched systems with time-varying delay," in Proceedings of 9th IEEE International Conference on Control and Automation, pp. 300-305, 2011.

[30] A. Iftar and E. J. Davison, "A decentralized discrete-time controller for dyrtamic routing," International Journal of Control, vol. 69, no. 5, pp. 599-632, 1998. 


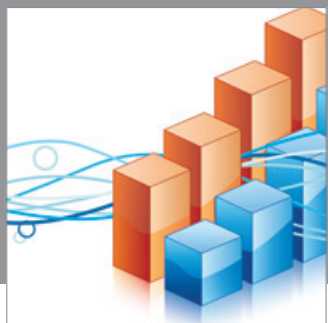

Advances in

Operations Research

mansans

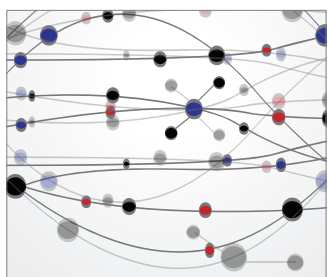

The Scientific World Journal
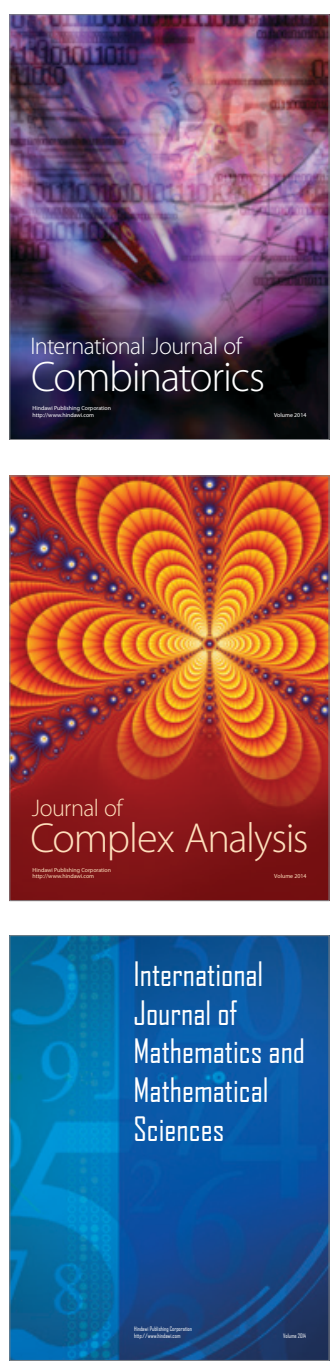
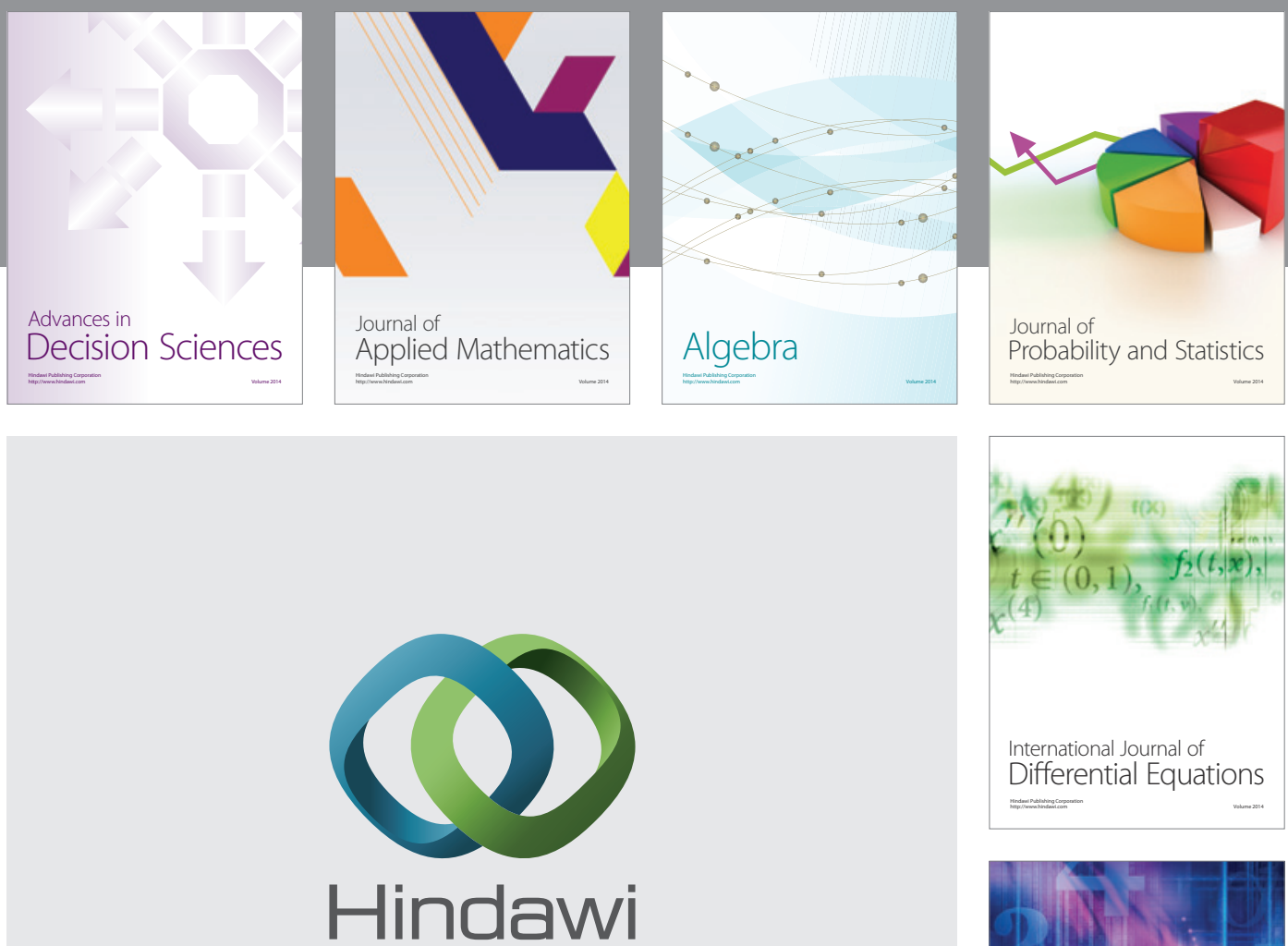

Submit your manuscripts at http://www.hindawi.com
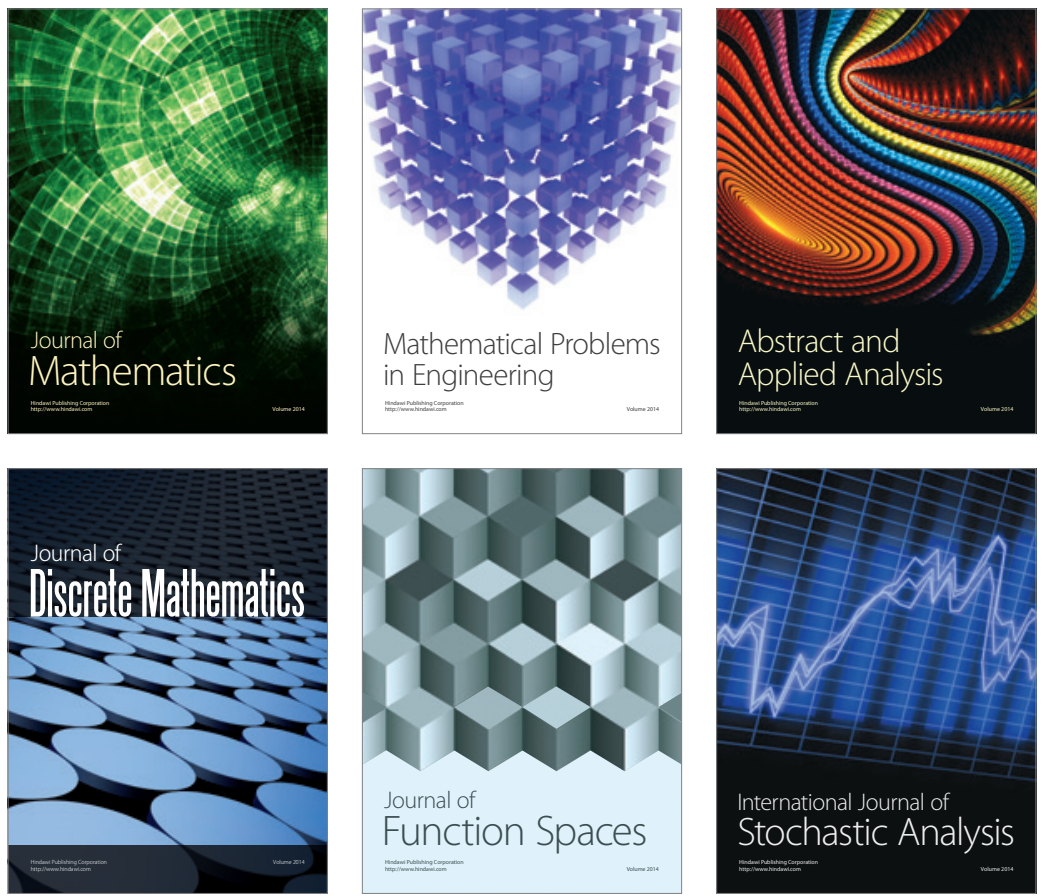

Journal of

Function Spaces

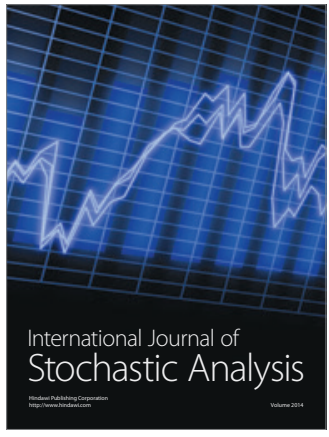

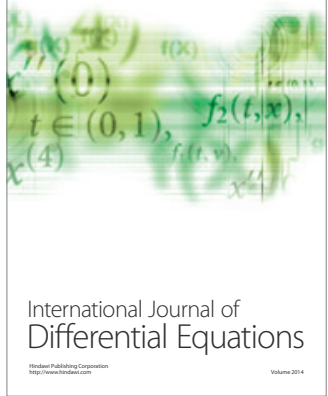
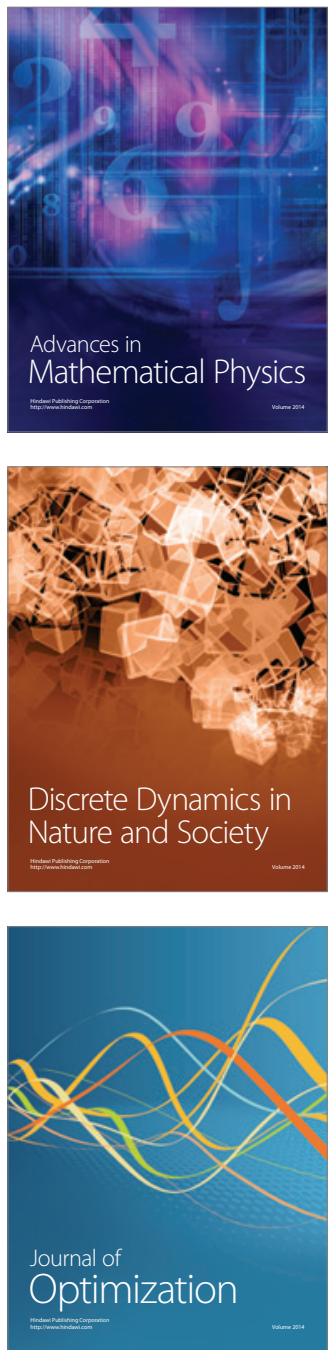